\title{
CHEMICAL COMPOSITION OF THE RS CVn-TYPE STAR LAMBDA ANDROMEDAE
}

\author{
G. Tautvaišienè ${ }^{1}$, G. Barisevičius ${ }^{1}$, S. Berdyugina ${ }^{2}$, Y. Chorniy ${ }^{1}$ and \\ I. Ilyin ${ }^{3}$ \\ 1 Institute of Theoretical Physics and Astronomy, Vilnius University, \\ Goštauto 12, Vilnius LT-01108, Lithuania \\ 2 Kiepenheuer Institut für Sonnenphysik, Schöneckstrasse 6, Freiburg D-79104, \\ Germany \\ 3 Astrophysical Institute Potsdam, An der Sternwarte 16, Potsdam D-14482, \\ Germany
}

Received: 2010 June 8; accepted: 2010 June 15

\begin{abstract}
Photospheric parameters and chemical composition are determined for the single-lined chromospherically active RS CVn-type star $\lambda$ And (HD 222107). From the high resolution spectra obtained on the Nordic Optical Telescope, abundances of 22 chemical elements and isotopes, including such key elements as ${ }^{12} \mathrm{C},{ }^{13} \mathrm{C}, \mathrm{N}$ and $\mathrm{O}$, were investigated. The differential line analysis with the MARCS model atmospheres gives $T_{\text {eff }}=4830 \mathrm{~K}, \log g=2.8,[\mathrm{Fe} / \mathrm{H}]$ $=-0.53,[\mathrm{C} / \mathrm{Fe}]=0.09,[\mathrm{~N} / \mathrm{Fe}]=0.35,[\mathrm{O} / \mathrm{Fe}]=0.45, \mathrm{C} / \mathrm{N}=2.21,{ }^{12} \mathrm{C} /{ }^{13} \mathrm{C}=$ 14. The ${ }^{12} \mathrm{C} /{ }^{13} \mathrm{C}$ ratio for a star of the $\mathrm{RS} C V n$-type is determined for the first time, and its low value gives a hint that extra-mixing processes may start acting in low-mass chromospherically active stars below the bump of the luminosity function of red giants.
\end{abstract}

Key words: stars: RS CVn binaries, abundances - stars: individual ( $\lambda$ And $=$ HD 222107)

\section{INTRODUCTION}

The RS CVn-type stars have been studied thoroughly since 1965 when their peculiar light curves were detected (Rodonó 1965; Chisari \& Lacona 1965) and a new distinct class of binaries was named (Olivier 1974; Hall 1976). RS CVn binary systems are typically composed of two late-type chromospherically active fast-rotating stars, at least one of which has already evolved off the main sequence (Hall 1976). Tidal forces between the close components make their rotational period to be equal to the orbital period. Similarly to other cool active stars, RS CVn-type variables are remarkable due to large starspots, strong chromospheric plages, coronal X-ray and microwave emissions, as well as strong flares in the optical, radio and other spectral regions. General properties of RS CVn systems are comprehensively described by Montesinos et al. (1988). The photometric brightness variation analysis, Doppler imaging and spectral line analysis of RS CVn stars indicate that starspots may cover more than $20 \%$ of their surfaces (Rodonó et al. 1995; Berdyugina et al. 1998b, 2000; Jeffers 2005; Alekseev \& Kozhevnikova 
2005). One of the largest filling factors (44\%) was found for the RS CVn binary V841 Cen (Strassmeier et al. 2008).

The photospheric abundances of chemical elements in RS CVn-type stars are found to be peculiar and indicate a combined action of various physical processes related to activity (e.g., Pallavicini et al. 1992, Tautvaišienè et al. 1992; Randich et al. 1993, 1994; Savanov \& Berdyugina 1994; Berdyugina et al. 1998a, 1999; Katz et al. 2003; Morel et al. 2003, 2004).

A new era of investigations of RS CVn stars has begun with the launch of the Extreme Ultraviolet Explorer, Chandra and XMM-Newton satellites and the study of element abundances in the coronal plasma. Highly active stars show a depletion of elements with a low first ionization potential (FIP) (e.g., $\mathrm{Fe}, \mathrm{Mg}, \mathrm{Si}$ ) relative to high-FIP elements (e.g., C, N, O, Ne), whereas active binaries with medium activity show either no the FIP feature or a possible solar-like FIP effect; some studies show that only low-FIP elements are sensitive to the activity level, while this is not the case for high-FIP elements (Drake 1996, 2002; Jordan et al. 1998; Audard et al. 2001, 2003; Bowyer et al. 2000; Sanz-Forcada et al. 2004 and references therein). In order to interpret the apparent coronal abundance anomalies and true abundance differences, a detailed comparison of photospheric abundances is needed.

We have started a detailed study of the photospheric abundances in RS CVn stars, and observed a sample of 28 such stars on the Nordic Optical Telescope. We consider that this study will be useful in addressing the issues of photospheric and coronal abundance patterns and mixing processes in these stars. Our aim is to determine abundances of more than 20 chemical elements, including ${ }^{12} \mathrm{C},{ }^{13} \mathrm{C}, \mathrm{N}$, $\mathrm{O}$ and other mixing-sensitive species. We plan to investigate correlations between abundance alterations of chemical elements in star atmospheres and their physical macro parameters, such as the speed of rotation and the magnetic field.

In this paper we present results of the analysis on one of the brightest RS CVn binaries, $\lambda$ And (HR 8961, HD 222107), consisting of a G8 III-IV $\operatorname{star}(V \approx 3.8 \mathrm{mag}$ ) and a spectroscopically undetected secondary. According to Donati et al. (1995), $\lambda$ And has a mass of $0.65_{-0.3}^{+0.6} M_{\odot}$, and the unseen secondary component of the system is a low-mass main-sequence star or a brown dwarf of mass $0.08 \pm 0.02 M_{\odot}$. Since the primary dominates the emission, the photospheric abundances can be relatively well determined, and thus the system is an ideal candidate for studying coronal-to-photospheric abundance patterns. According to Savanov \& Berdyugina (1994), the mass of $\lambda$ And primary is $1.2 M_{\odot}$.

Calder (1938) was the first to discover the photometric variability of $\lambda$ And, its amplitude sometimes reaches 0.3 mag. Six years later, Walker (1944) showed that $\lambda$ And is an SB1 with almost circular orbit of period $20.5212 \mathrm{~d}$. It is atypical for a member of RS CVn type stars, because it is considerably out of synchronism, its rotational period being $54.3 \mathrm{~d}$ (Gondoin 2007). In most RS CVn binaries the rotational period of both components is equal, within a few percent, to the orbital period of the system. Thus $\lambda$ And, whose orbit is very close to circular, is a puzzle for the theory of tidal friction (Zahn 1977), which predicts that rotational synchronization in close binaries should precede circulization of the orbit.

From more than 26 years of photometry, Mirtorabi et al. (2003) found that the mean light level of $\lambda$ And varies between $\langle V\rangle_{\min }=3.89 \mathrm{mag}$ and $\langle V\rangle_{\max }=$ 3.77 mag. These observations have shown that $\lambda$ And exhibits semi-regular cyclic light variations between $4-5 \mathrm{yr}$ and $\sim 14 \mathrm{yr}$. From these data, well-defined light 
minima were noticed in 1979/1980, 1991 and 1997, and well-defined light maxima in 1985/1986 and 1999/2000. Our observations of $\lambda$ And have been done during its light maximum in August of 1999.

For $\lambda$ And, the asymmetrical shape of the light curve requires two spots at different longitudes and these spots must be $800-1050 \mathrm{~K}$ cooler than the surrounding photosphere (Bopp \& Noah 1980; Poe \& Eaton 1985; Donati et al. 1995; Padmakar \& Pandey 1999; Frasca et al. 2008). According to O'Neal et al. (1998), the dark spot filling factor for $\lambda$ And was 0.14 in late summer of 1993 and even 0.23 in January of 1995. According to Frasca et al. (2008), the star radius is $R=7.51 R_{\odot}$ and the inclination of the rotational axis with respect to the line of sight is $i=67^{\circ}$. Donati et al. (1995) give $v \sin i=6.5 \mathrm{~km} \mathrm{~s}^{-1}$.

The investigation of possible dependencies of spot parameters, such as the temperature and filling factor, on global stellar parameters, such as the effective temperature, gravity and activity level (rotation rate, differential rotation, etc.), is very important to better understand the physical mechanisms at work in the formation and evolution of RS CVn stars.

The correlation of the temperature difference between the quiet photosphere and spots, $\Delta T$, with the effective temperature has been determined by Berdyugina (2005). On the average, $\Delta T$ is larger for the hotter stars, with the values from about $2000 \mathrm{~K}$ for G-type stars to $200 \mathrm{~K}$ for M4 stars. This behavior is displayed both for giants and main-sequence stars. It is interesting to investigate the role of the surface gravity on $\Delta T$ by selecting stars of nearly the same temperature.

\section{OBSERVATIONS AND THE METHOD OF ANALYSIS}

The spectra were obtained in August of 1999 on the 2.56 m Nordic Optical Telescope using the SOFIN echelle spectrograph with the optical camera, which provided a spectral resolving power of $R \approx 80000$, for 26 spectral orders, each of $\sim 40 \AA$, in the spectral region $500-830 \mathrm{~nm}$. Reductions of the CCD images were made with the ' $4 \mathrm{~A}$ ' software package (Ilyin 2000). In image processing, the procedures of bias subtraction, spike elimination, flat field correction, scattered light subtraction and extraction of spectral orders were used. The continuum was defined by a number of narrow spectral regions, selected to be free of lines. In the spectra of $\lambda$ And, we selected 118 atomic lines for the measurement of equivalent widths and 19 lines for the comparison with synthetic spectra. The equivalent widths were measured by fitting a Gaussian function with the ' $4 \mathrm{~A}$ ' software package (Ilyin 2000). The measured equivalent widths of the lines and their parameters are presented in Table 1.

The spectra were analysed using a differential model atmosphere technique. The EQWIDTH and BSYN program packages, developed at the Uppsala Astronomical Observatory, were used to carry out the calculations of theoretical equivalent widths of lines and synthetic spectra. First, using the EQWIDTH program package we obtained solar abundances which were later used for the differential determination of abundances of the program stars. The solar model atmosphere was taken from the Uppsala set (Gustafsson et al. 2008) with $T_{\text {eff }}=5777 \mathrm{~K}$, $\log g=4.44$ and $v_{\mathrm{t}}=0.8 \mathrm{~km} \mathrm{~s}^{-1}$ determined from the Fe I lines. 
Table 1. Atomic line data and the measured equivalent widths. In the head of the table $\chi_{l}$ is the excitation potential of the lower level, $\log g f$ is the oscillator strength, $\delta \Gamma_{6}$ is the correction factor to the van-der-Waals damping constant, $\Gamma_{\text {rad }}$ is the radiation damping constant, $E W_{\star}$ and $E W_{\odot}$ are the equivalent widths of lines in $\lambda$ And and in the solar spectrum.

\begin{tabular}{|c|c|c|c|c|c|c|c|}
\hline Element & $\lambda(\AA)$ & $\chi_{l}(\mathrm{eV})$ & $\log g f$ & $\delta \Gamma_{6}$ & $\Gamma_{\mathrm{rad}}\left(\mathrm{s}^{-1}\right)$ & $E W_{\star}(\mathrm{m} \AA)$ & $E W_{\odot}(\mathrm{m} \AA)$ \\
\hline \multirow[t]{2}{*}{ Si I } & 5793.08 & 4.93 & -1.98 & 1.30 & $1.95 \mathrm{e}+08$ & 44.3 & 43.5 \\
\hline & 6131.85 & 5.61 & -1.72 & 1.30 & $1.00 \mathrm{e}+05$ & 22.6 & 25.7 \\
\hline \multirow[t]{3}{*}{ Ca I } & 5867.57 & 2.93 & -1.59 & 1.80 & $2.62 \mathrm{e}+08$ & 49.0 & 23.9 \\
\hline & 6455.60 & 2.52 & -1.42 & 1.80 & $4.65 \mathrm{e}+07$ & 93.0 & 56.7 \\
\hline & 6798.47 & 2.71 & -2.49 & 1.80 & $1.94 \mathrm{e}+07$ & 22.5 & 6.3 \\
\hline \multirow[t]{2}{*}{ Sc II } & 5526.81 & 1.77 & 0.25 & 2.50 & $2.16 \mathrm{e}+08$ & 109.5 & 78.7 \\
\hline & 6300.69 & 1.51 & -1.93 & 2.50 & $2.31 \mathrm{e}+08$ & 15.6 & 5.6 \\
\hline \multirow[t]{16}{*}{ Ti I } & 5648.58 & 2.49 & -0.35 & 2.50 & $5.06 \mathrm{e}+07$ & 44.8 & 10.1 \\
\hline & 5662.16 & 2.32 & -0.14 & 2.50 & $6.03 \mathrm{e}+07$ & 71.7 & 20.8 \\
\hline & 5716.45 & 2.30 & -0.82 & 2.50 & $6.05 \mathrm{e}+07$ & 31.2 & 5.8 \\
\hline & 5739.48 & 2.25 & -0.69 & 2.50 & $6.61 \mathrm{e}+07$ & 41.1 & 8.1 \\
\hline & 5899.30 & 1.05 & -1.19 & 2.50 & $6.58 \mathrm{e}+07$ & 103.4 & 28.8 \\
\hline & 5903.31 & 1.07 & -2.10 & 2.50 & $6.46 \mathrm{e}+06$ & 43.2 & 4.6 \\
\hline & 5941.76 & 1.05 & -1.55 & 2.50 & $7.13 \mathrm{e}+07$ & 80.1 & 15.1 \\
\hline & 5953.17 & 1.89 & -0.33 & 2.50 & $8.61 \mathrm{e}+06$ & 90.1 & 31.3 \\
\hline & 5965.83 & 1.88 & -0.46 & 2.50 & $8.91 \mathrm{e}+06$ & 83.6 & 25.5 \\
\hline & 6064.63 & 1.05 & -1.86 & 2.50 & $9.93 \mathrm{e}+06$ & 59.5 & 8.1 \\
\hline & 6098.66 & 3.06 & -0.14 & 2.50 & $5.43 \mathrm{e}+07$ & 22.0 & 5.3 \\
\hline & 6121.00 & 1.88 & -1.45 & 2.50 & $1.23 \mathrm{e}+08$ & 28.4 & 3.5 \\
\hline & 6126.22 & 1.07 & -1.37 & 2.50 & $9.93 \mathrm{e}+06$ & 86.1 & 21.0 \\
\hline & 6220.49 & 2.68 & -0.29 & 2.50 & $4.61 \mathrm{e}+07$ & 39.5 & 8.4 \\
\hline & 6303.77 & 1.44 & -1.54 & 2.50 & $1.75 \mathrm{e}+08$ & 51.8 & 7.4 \\
\hline & 6599.11 & 0.90 & -2.05 & 2.50 & $1.22 \mathrm{e}+06$ & 66.9 & 8.0 \\
\hline \multirow[t]{14}{*}{$\mathrm{VI}_{\mathrm{I}}$} & 5604.96 & 1.04 & -1.17 & 2.50 & $8.47 \mathrm{e}+07$ & 33.5 & 3.6 \\
\hline & 5646.11 & 1.05 & -1.10 & 2.50 & $8.53 \mathrm{e}+07$ & 36.4 & 4.1 \\
\hline & 5657.45 & 1.06 & -1.01 & 2.50 & $8.47 \mathrm{e}+07$ & 45.7 & 5.0 \\
\hline & 5668.37 & 1.08 & -0.93 & 2.50 & $8.39 \mathrm{e}+07$ & 43.5 & 5.7 \\
\hline & 5743.43 & 1.08 & -0.88 & 2.50 & $7.67 \mathrm{e}+07$ & 51.8 & 6.3 \\
\hline & 6058.18 & 1.04 & -1.36 & 2.50 & $3.94 \mathrm{e}+07$ & 29.9 & 2.5 \\
\hline & 6111.65 & 1.04 & -0.68 & 2.50 & $3.90 \mathrm{e}+07$ & 71.5 & 11.0 \\
\hline & 6119.53 & 1.06 & -0.41 & 2.50 & $3.94 \mathrm{e}+07$ & 82.5 & 18.0 \\
\hline & 6135.37 & 1.05 & -0.73 & 2.50 & $3.90 \mathrm{e}+07$ & 63.5 & 9.7 \\
\hline & 6224.50 & 0.29 & -1.79 & 2.50 & $1.22 \mathrm{e}+06$ & 60.8 & 5.0 \\
\hline & 6233.19 & 0.28 & -1.90 & 2.50 & $1.35 \mathrm{e}+06$ & 53.8 & 4.0 \\
\hline & 6266.30 & 0.28 & -2.08 & 2.50 & $1.37 \mathrm{e}+06$ & 43.2 & 2.6 \\
\hline & 6274.66 & 0.27 & -1.64 & 2.50 & $3.10 \mathrm{e}+06$ & 61.9 & 7.3 \\
\hline & 6285.16 & 0.28 & -1.53 & 2.50 & $2.91 \mathrm{e}+06$ & 71.9 & 8.9 \\
\hline \multirow[t]{5}{*}{ Cr I } & 5712.78 & 3.00 & -1.11 & 2.50 & $1.78 \mathrm{e}+08$ & 26.4 & 13.9 \\
\hline & 5783.87 & 3.32 & -0.21 & 2.50 & $9.98 \mathrm{e}+07$ & 62.8 & 39.0 \\
\hline & 5784.97 & 3.32 & -0.39 & 2.50 & $9.98 \mathrm{e}+07$ & 46.7 & 29.9 \\
\hline & 5787.92 & 3.32 & -0.06 & 2.50 & $1.01 \mathrm{e}+08$ & 62.8 & 47.2 \\
\hline & 6979.80 & 3.46 & -0.22 & 2.50 & $2.53 \mathrm{e}+08$ & 54.8 & 35.0 \\
\hline \multirow[t]{8}{*}{$\mathrm{Fe} I$} & 5406.78 & 4.37 & -1.46 & 1.40 & $1.77 \mathrm{e}+08$ & 43.1 & 38.5 \\
\hline & 5522.45 & 4.21 & -1.52 & 1.40 & $8.97 \mathrm{e}+07$ & 51.4 & 43.1 \\
\hline & 5577.03 & 5.03 & -1.54 & 1.40 & $6.89 \mathrm{e}+08$ & 11.6 & 12.1 \\
\hline & 5651.48 & 4.47 & -1.87 & 1.40 & $1.63 \mathrm{e}+08$ & 21.4 & 17.4 \\
\hline & 5652.33 & 4.26 & -1.83 & 1.40 & $8.18 \mathrm{e}+07$ & 31.0 & 26.2 \\
\hline & 5653.86 & 4.39 & -1.49 & 1.40 & $1.79 \mathrm{e}+08$ & 42.9 & 36.9 \\
\hline & 5679.03 & 4.65 & -0.75 & 1.40 & $1.40 \mathrm{e}+08$ & 66.8 & 64.6 \\
\hline & 5720.90 & 4.55 & -1.88 & 1.40 & $1.92 \mathrm{e}+08$ & 18.7 & 15.0 \\
\hline
\end{tabular}


Table 1. Continued

\begin{tabular}{|c|c|c|c|c|c|c|c|}
\hline Element & $\lambda(\AA)$ & $\chi_{l}(\mathrm{eV})$ & $\log g f$ & $\delta \Gamma_{6}$ & $\Gamma_{\mathrm{rad}}\left(\mathrm{s}^{-1}\right)$ & $E W_{\star}(\mathrm{m} \AA)$ & $E W_{\odot}(\mathrm{m} \AA)$ \\
\hline Fe I & $\begin{array}{l}5732.30 \\
5738.24 \\
5741.86 \\
5784.67 \\
5793.92 \\
5806.73 \\
5809.22 \\
5811.92 \\
5814.82 \\
6027.06 \\
6034.04 \\
6035.35 \\
6054.07 \\
6056.01 \\
6098.25 \\
6105.13 \\
6120.24 \\
6187.99 \\
6226.74 \\
6229.23 \\
6270.23 \\
6380.75 \\
6392.54 \\
6574.21 \\
6581.21 \\
6646.97 \\
6786.86 \\
6793.27 \\
6839.83 \\
6842.69 \\
6843.65 \\
6851.64 \\
6857.25 \\
6858.15 \\
6862.49 \\
7461.53\end{array}$ & $\begin{array}{l}4.99 \\
4.22 \\
4.26 \\
3.40 \\
4.22 \\
4.61 \\
3.88 \\
4.14 \\
4.28 \\
4.07 \\
4.31 \\
4.29 \\
4.37 \\
4.73 \\
4.56 \\
4.55 \\
0.91 \\
3.94 \\
3.88 \\
2.84 \\
2.86 \\
4.19 \\
2.28 \\
0.99 \\
1.48 \\
2.61 \\
4.19 \\
4.07 \\
2.56 \\
4.64 \\
4.55 \\
1.61 \\
4.07 \\
4.61 \\
4.56 \\
2.56\end{array}$ & $\begin{array}{l}-1.54 \\
-2.29 \\
-1.73 \\
-2.69 \\
-1.69 \\
-0.96 \\
-1.73 \\
-2.45 \\
-1.90 \\
-1.23 \\
-2.43 \\
-2.60 \\
-2.29 \\
-0.47 \\
-1.88 \\
-2.09 \\
-5.91 \\
-1.68 \\
-2.16 \\
-3.04 \\
-2.64 \\
-1.39 \\
-4.05 \\
-5.03 \\
-4.75 \\
-3.97 \\
-1.99 \\
-2.48 \\
-3.43 \\
-1.25 \\
-0.90 \\
-5.39 \\
-2.17 \\
-1.01 \\
-1.52 \\
-3.57\end{array}$ & $\begin{array}{l}1.40 \\
1.40 \\
1.40 \\
1.40 \\
1.40 \\
1.40 \\
1.40 \\
1.40 \\
1.40 \\
1.40 \\
1.40 \\
1.40 \\
1.40 \\
1.40 \\
1.40 \\
1.40 \\
1.40 \\
1.40 \\
1.40 \\
1.40 \\
1.40 \\
1.40 \\
1.40 \\
1.40 \\
1.40 \\
1.40 \\
1.40 \\
1.40 \\
1.40 \\
1.40 \\
1.40 \\
1.40 \\
1.40 \\
1.40 \\
1.40 \\
1.40\end{array}$ & $\begin{array}{l}6.89 \mathrm{e}+08 \\
2.01 \mathrm{e}+08 \\
2.10 \mathrm{e}+08 \\
7.53 \mathrm{e}+07 \\
2.11 \mathrm{e}+08 \\
1.86 \mathrm{e}+08 \\
5.06 \mathrm{e}+07 \\
3.76 \mathrm{e}+07 \\
2.11 \mathrm{e}+08 \\
8.85 \mathrm{e}+07 \\
1.58 \mathrm{e}+08 \\
6.92 \mathrm{e}+07 \\
1.66 \mathrm{e}+08 \\
1.85 \mathrm{e}+08 \\
2.62 \mathrm{e}+08 \\
1.94 \mathrm{e}+08 \\
2.71 \mathrm{e}+04 \\
4.60 \mathrm{e}+07 \\
5.42 \mathrm{e}+07 \\
1.45 \mathrm{e}+08 \\
1.45 \mathrm{e}+08 \\
7.35 \mathrm{e}+07 \\
1.65 \mathrm{e}+08 \\
3.38 \mathrm{e}+04 \\
1.56 \mathrm{e}+07 \\
7.57 \mathrm{e}+07 \\
1.99 \mathrm{e}+08 \\
7.08 \mathrm{e}+07 \\
9.31 \mathrm{e}+07 \\
2.23 \mathrm{e}+08 \\
1.92 \mathrm{e}+08 \\
1.46 \mathrm{e}+07 \\
2.53 \mathrm{e}+07 \\
1.92 \mathrm{e}+08 \\
3.54 \mathrm{e}+08 \\
1.21 \mathrm{e}+08\end{array}$ & $\begin{array}{r}11.8 \\
17.2 \\
37.7 \\
41.0 \\
40.9 \\
57.2 \\
61.9 \\
12.9 \\
26.7 \\
78.9 \\
10.7 \\
7.3 \\
12.2 \\
74.6 \\
15.7 \\
12.1 \\
27.2 \\
61.0 \\
36.7 \\
59.4 \\
80.2 \\
61.0 \\
37.1 \\
76.4 \\
57.6 \\
21.9 \\
27.6 \\
18.0 \\
55.9 \\
40.9 \\
67.0 \\
15.9 \\
26.5 \\
56.1 \\
33.3 \\
55.6\end{array}$ & $\begin{array}{r}13.1 \\
12.2 \\
31.2 \\
24.3 \\
35.0 \\
54.8 \\
48.6 \\
10.3 \\
22.8 \\
66.4 \\
7.9 \\
5.8 \\
9.4 \\
78.8 \\
15.1 \\
10.2 \\
5.0 \\
49.9 \\
28.8 \\
33.6 \\
53.2 \\
53.6 \\
15.4 \\
26.2 \\
18.4 \\
9.8 \\
24.6 \\
12.1 \\
29.8 \\
40.1 \\
64.3 \\
3.9 \\
22.3 \\
55.2 \\
30.7 \\
25.5\end{array}$ \\
\hline Fe II & $\begin{array}{l}5256.93 \\
5264.81 \\
5534.84 \\
6113.33 \\
6369.46 \\
6383.71 \\
6456.39 \\
7711.72\end{array}$ & $\begin{array}{l}2.88 \\
3.22 \\
3.24 \\
3.22 \\
2.89 \\
5.55 \\
3.90 \\
3.90\end{array}$ & $\begin{array}{l}-4.25 \\
-3.26 \\
-2.98 \\
-4.26 \\
-4.28 \\
-2.25 \\
-2.28 \\
-2.72\end{array}$ & $\begin{array}{l}2.50 \\
2.50 \\
2.50 \\
2.50 \\
2.50 \\
2.50 \\
2.50 \\
2.50\end{array}$ & $\begin{array}{l}3.41 \mathrm{e}+08 \\
4.11 \mathrm{e}+08 \\
2.99 \mathrm{e}+08 \\
3.41 \mathrm{e}+08 \\
2.90 \mathrm{e}+08 \\
4.09 \mathrm{e}+08 \\
3.37 \mathrm{e}+08 \\
4.12 \mathrm{e}+08\end{array}$ & \begin{tabular}{r|}
21.3 \\
40.2 \\
56.6 \\
9.9 \\
15.9 \\
5.1 \\
52.3 \\
34.3
\end{tabular} & $\begin{array}{l}19.9 \\
47.6 \\
60.0 \\
12.0 \\
20.1 \\
10.9 \\
66.1 \\
49.8\end{array}$ \\
\hline $\mathrm{CoI}$ & $\begin{array}{l}5647.23 \\
6595.86 \\
6678.82\end{array}$ & $\begin{array}{l}2.28 \\
3.71 \\
1.96\end{array}$ & $\begin{array}{l}-1.51 \\
-0.64 \\
-2.18\end{array}$ & $\begin{array}{l}2.50 \\
2.50 \\
2.50\end{array}$ & $\begin{array}{l}1.66 \mathrm{e}+08 \\
6.84 \mathrm{e}+07 \\
2.01 \mathrm{e}+07\end{array}$ & $\begin{array}{l}37.6 \\
10.7 \\
22.5\end{array}$ & $\begin{array}{r}13.3 \\
5.0 \\
6.8\end{array}$ \\
\hline $\mathrm{Ni}$ I & $\begin{array}{l}5578.73 \\
5587.87 \\
5589.37 \\
5593.75 \\
5643.09 \\
5748.35 \\
5805.22 \\
6111.08 \\
6128.98\end{array}$ & $\begin{array}{l}1.68 \\
1.93 \\
3.90 \\
3.90 \\
4.16 \\
1.68 \\
4.17 \\
4.09 \\
1.68\end{array}$ & $\begin{array}{l}-2.56 \\
-2.28 \\
-1.03 \\
-0.67 \\
-1.13 \\
-3.12 \\
-0.48 \\
-0.72 \\
-3.27\end{array}$ & $\begin{array}{l}2.50 \\
2.50 \\
2.50 \\
2.50 \\
2.50 \\
2.50 \\
2.50 \\
2.50 \\
2.50\end{array}$ & $\begin{array}{l}5.43 \mathrm{e}+07 \\
1.25 \mathrm{e}+08 \\
1.30 \mathrm{e}+08 \\
1.32 \mathrm{e}+08 \\
9.14 \mathrm{e}+07 \\
4.31 \mathrm{e}+07 \\
2.17 \mathrm{e}+08 \\
1.46 \mathrm{e}+08 \\
1.21 \mathrm{e}+07\end{array}$ & $\begin{array}{l}90.7 \\
86.0 \\
31.6 \\
46.4 \\
15.6 \\
59.3 \\
41.5 \\
36.4 \\
56.8\end{array}$ & $\begin{array}{l}55.0 \\
56.9 \\
27.9 \\
45.2 \\
15.3 \\
29.0 \\
43.0 \\
35.5 \\
23.6\end{array}$ \\
\hline
\end{tabular}


Table 1. Continued

\begin{tabular}{lccccccc}
\hline Element & $\lambda(\AA)$ & $\chi_{l}(\mathrm{eV})$ & $\log g f$ & $\delta \Gamma_{6}$ & $\Gamma_{\operatorname{rad}}\left(\mathrm{s}^{-1}\right)$ & $E W_{\star}(\mathrm{m} \AA)$ & $E W_{\odot}(\mathrm{m} \AA)$ \\
\hline \multirow{2}{*}{ Ni I } & 6130.14 & 4.26 & -0.89 & 2.50 & $2.78 \mathrm{e}+08$ & 20.0 & 21.0 \\
& 6378.25 & 4.15 & -0.76 & 2.50 & $2.08 \mathrm{e}+08$ & 35.2 & 31.5 \\
& 6598.60 & 4.23 & -0.85 & 2.50 & $1.92 \mathrm{e}+08$ & 26.7 & 24.5 \\
& 6635.13 & 4.42 & -0.68 & 2.50 & $1.51 \mathrm{e}+08$ & 22.4 & 24.7 \\
& 6772.32 & 3.66 & -0.86 & 2.50 & $1.50 \mathrm{e}+08$ & 56.5 & 50.9 \\
& 6842.03 & 3.66 & -1.36 & 2.50 & $1.51 \mathrm{e}+08$ & 33.0 & 26.1 \\
& 7001.55 & 1.93 & -3.48 & 2.50 & $4.86 \mathrm{e}+07$ & 30.4 & 11.1 \\
& 7715.59 & 3.70 & -0.87 & 2.50 & $5.87 \mathrm{e}+07$ & 58.5 & 52.0 \\
\hline
\end{tabular}

The atomic oscillator strengths and solar equivalent widths for the lines (Table 1) were taken from Gurtovenko \& Kostik (1989). The Vienna Atomic Line Data Base (VALD) (Piskunov et al. 1995) was used in preparing other input data for the calculations. In addition to thermal and microturbulent Doppler broadening of lines, atomic line broadening by radiation damping and van der Waals damping were considered in the calculation of abundances. In most cases the hydrogen pressure damping of metal lines was treated using the modern quantum mechanical calculations by Anstee \& O'Mara (1995), Barklem \& O'Mara (1997) and Barklem et al. (1998). When using the Unsöld (1955) approximation, correction factors to the classical van der Waals damping approximation by widths $\left(\Gamma_{6}\right)$ were taken from Simmons \& Blackwell (1982). For all other species a correction factor of 2.5 was applied to the classical $\Gamma_{6}\left(\Delta \log C_{6}=+1.0\right)$, following Mäckle et al. (1975).

\subsection{Atmospheric parameters}

Initially, the effective temperature $T_{\text {eff }}$ of $\lambda$ And was derived and averaged from the intrinsic color indices $(B-V)_{0}$ and $(b-y)_{0}$ using corresponding calibrations by Alonso et al. (1999). The color indices $B-V=0.984$ and $b-y=0.625$ were taken from van Leeuwen et al. (2007) and Hauck \& Mermilliod (1998), respectively. A small dereddening correction of $E_{B-V}=0.01$, estimated by the Hakkila et al. (1997) software, was taken into account.

The agreement between the temperatures deduced from the two color indices was quite good, the difference was only $20 \mathrm{~K}$. No obvious trend of the Fe I abundances with the excitation potential was found (Figure 1). The surface gravity $\log g$ was found by adjusting the model gravity to yield the same iron abundance from the FeI and Fe II lines. Microturbulent velocity $v_{\mathrm{t}}$ value corresponding to minimal line-to-line Fe I abundance scattering was chosen as the correct value. Consequently, the $[\mathrm{Fe} / \mathrm{H}]$ values do not depend on the equivalent widths of lines (Figure 2).

\subsection{Mass determination}

The mass of the $\lambda$ And was evaluated from its effective temperature, luminosity and the Girardi et al. (2000) isochrones. The luminosity $\log \left(L / L_{\odot}\right)=1.37$ was calculated from the Hipparcos parallax $\pi=37.87$ mas (van Leeuwen 2007), $\langle V\rangle_{\max }$ $=3.77 \mathrm{mag}$ (Mirtorabi et al. 2003), the bolometric correction calculated according to Alonso et al. (1999) and $E_{B-V}=0.01$. The mass of $\lambda$ And is found to be $1.1 M_{\odot}$. This value is close to the mass $1.2 M_{\odot}$ determined by Savanov \& Berdyugina (1994). 


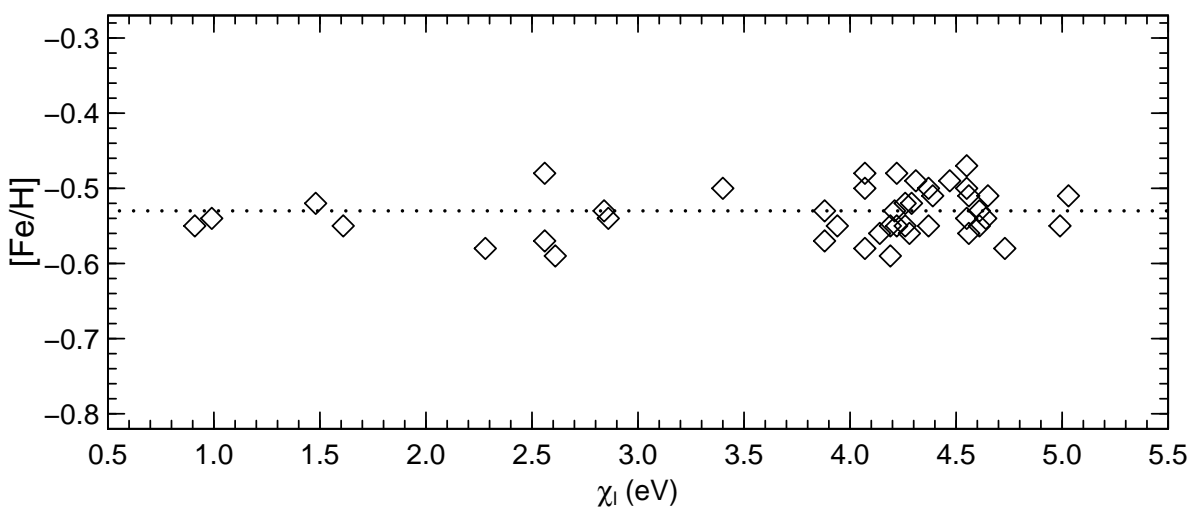

Fig. 1. The $[\mathrm{Fe} / \mathrm{H}]$ abundance values versus the lower excitation potential $\chi_{\text {exc }}$ for $\lambda$ And. The mean abundance $([\mathrm{Fe} / \mathrm{I}]=-0.53 \mathrm{dex})$ is shown by a dotted line.

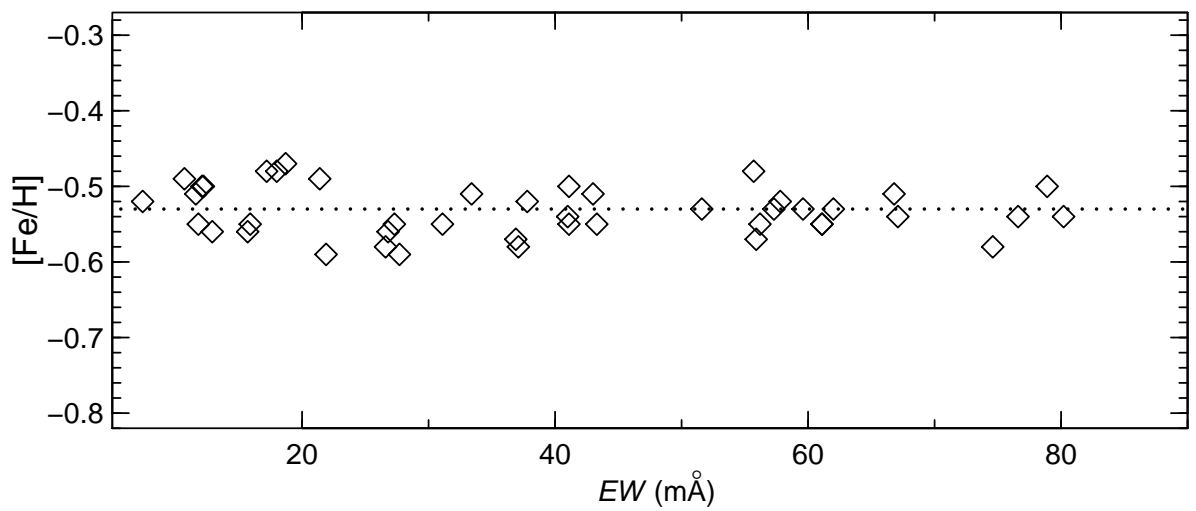

Fig. 2. [The $\mathrm{Fe} / \mathrm{H}]$ abundance values versus the equivalent widths for $\lambda$ And. The mean abundance $([\mathrm{Fe} \mathrm{I} / \mathrm{H}]=-0.53 \mathrm{dex})$ is shown by a dotted line.

\subsection{Synthetic spectra}

The method of synthetic spectra was used to determine the carbon abundance from the $\mathrm{C}_{2}$ line at $5135.5 \AA$ using the the Gonzalez et al. (1998) molecular data.

The interval $798-813 \AA$, containing strong ${ }^{12} \mathrm{C}^{14} \mathrm{~N}$ and ${ }^{13} \mathrm{C}^{14} \mathrm{~N}$ features, was used for determining the nitrogen abundance and the ${ }^{12} \mathrm{C} /{ }^{13} \mathrm{C}$ ratio. The molecular data for this band were provided by Bertrand Plez (University of Montpellier II). The ${ }^{12} \mathrm{C} /{ }^{13} \mathrm{C}$ ratio was determined from $(2,0){ }^{13} \mathrm{C}^{12} \mathrm{~N}$ feature at $8004.7 \AA$. All $\log g f$ values were calibrated to fit to the solar spectrum by Kurucz (2005) with solar abundances from Grevesse \& Sauval (2000). In Figure 3 we show several examples of synthetic spectra in the vicinity of ${ }^{12} \mathrm{C}^{14} \mathrm{~N}$ lines. 


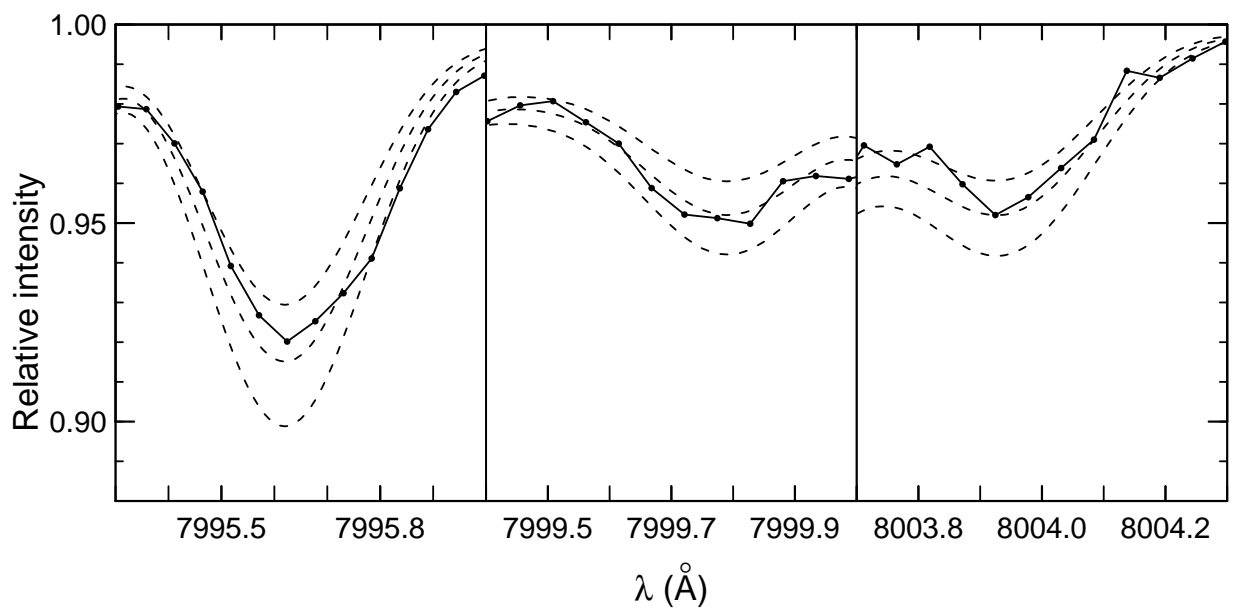

Fig. 3. Synthetic spectrum fits to the three ${ }^{12} \mathrm{CN}$ lines for $\lambda$ And. The observed spectra are shown as solid lines joining the dots. The dashed lines denote the synthetic spectra with $[\mathrm{N} / \mathrm{Fe}]=0.25,0.35$ and 0.45 dex downward.

The oxygen abundance was determined from the forbidden [O I] line at $6300.31 \AA$ (Figure 4) with the oscillator strengths for ${ }^{58} \mathrm{Ni}$ and ${ }^{60} \mathrm{Ni}$ from Johansson et al. (2003), the $\log g f=-9.917$ value, obtained by fitting to the solar spectrum of Kurucz (2005) and $\log A_{\odot}=8.83$ taken from Grevesse \& Sauval (2000).

The abundance of Na I was estimated using the line $5148.84 \AA$ which due to rotational broadening is blended by the Ni I line at $5148.66 \AA$. These two lines are distinct in the Sun, so we were able to calibrate their $\log g f$ values using the solar spectrum. However, the sodium abundance value in $\lambda$ And is affected by uncertainty of nickel abundance determination, originating from the Equivalent Widths method. Fortunately, the line-to-line scatter of $[\mathrm{Ni} / \mathrm{H}]$ determinations from 19 lines of Ni I was as small as 0.04 dex.

For the evaluation of $\mathrm{Zr}$ I abundance the lines at $5385.13 \AA, 6127.48 \AA$ and $6134.57 \AA$ were used. Evaluation of Y II abundance (Figure 5) was based on $5402.78 \AA$, Pr II on $5259.72 \AA$, La II on $6390.48 \AA$, Ce II on $5274.22 \AA$ and $6043.38 \AA$, and Nd II on $5276.86 \AA$ lines.

The abundance of Eu II was determined from the 6645.10 A line (Figure 6). The hyperfine structure of Eu II was taken into account when calculating the synthetic spectrum. The wavelength, excitation energy and total $\log g f=0.12$ were taken from Lawler et al. (2001), the isotopic meteoritic fractions of ${ }^{151} \mathrm{Eu}, 47.77 \%$, and ${ }^{153} \mathrm{Eu}, 52.23 \%$, and isotopic shifts were taken from Biehl (1976).

Due to the rotation of RS CVn stars, their lines are broadened, so it is important to use a correct value of $v \sin i$ in the synthetic spectrum production. We used the most recent value, $v \sin i=6.9 \mathrm{kms}^{-1}$, taken from De Medeiros et al. (2002), which fits our data quite well. Previous authors provided higher values of $v \sin i$, up to $20 \mathrm{~km} \mathrm{~s}^{-1}$ (Uesugi \& Fukuda 1982), which seem to be overestimated. 


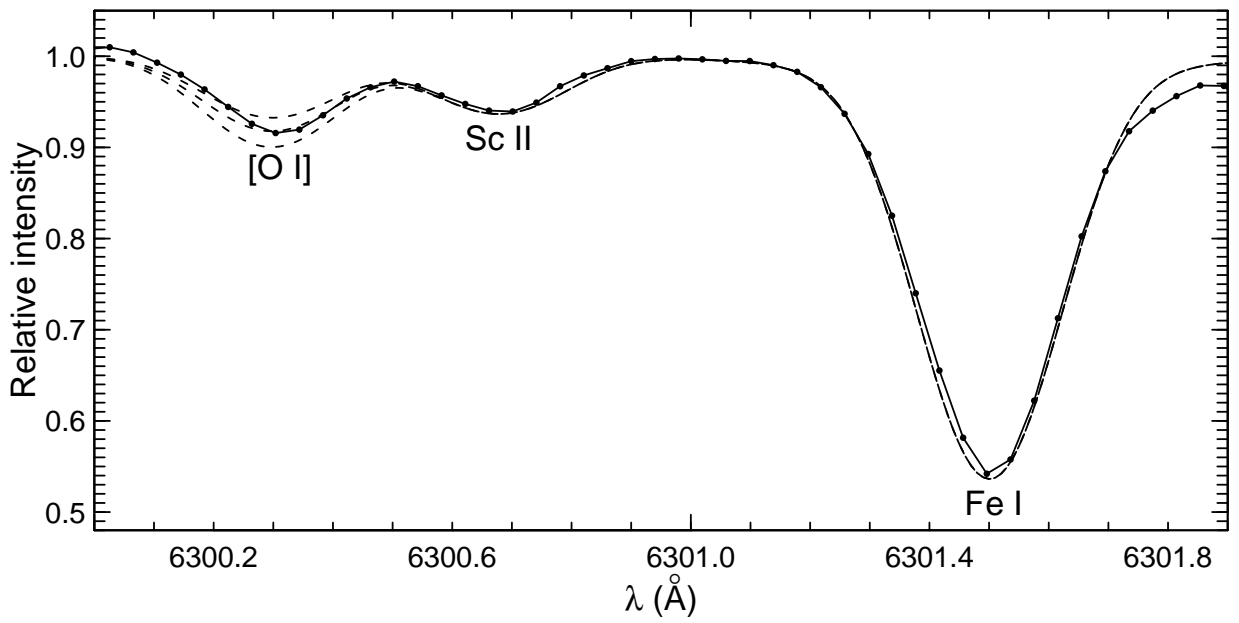

Fig. 4. Synthetic spectrum fit to the forbidden [O I] line at $6300 \AA$ for $\lambda$ And. The observed spectrum is shown as the solid line joining the dots. The dashed lines denote the synthetic spectra with $[\mathrm{O} / \mathrm{Fe}]=0.35,0.45$ and 0.55 dex downward.

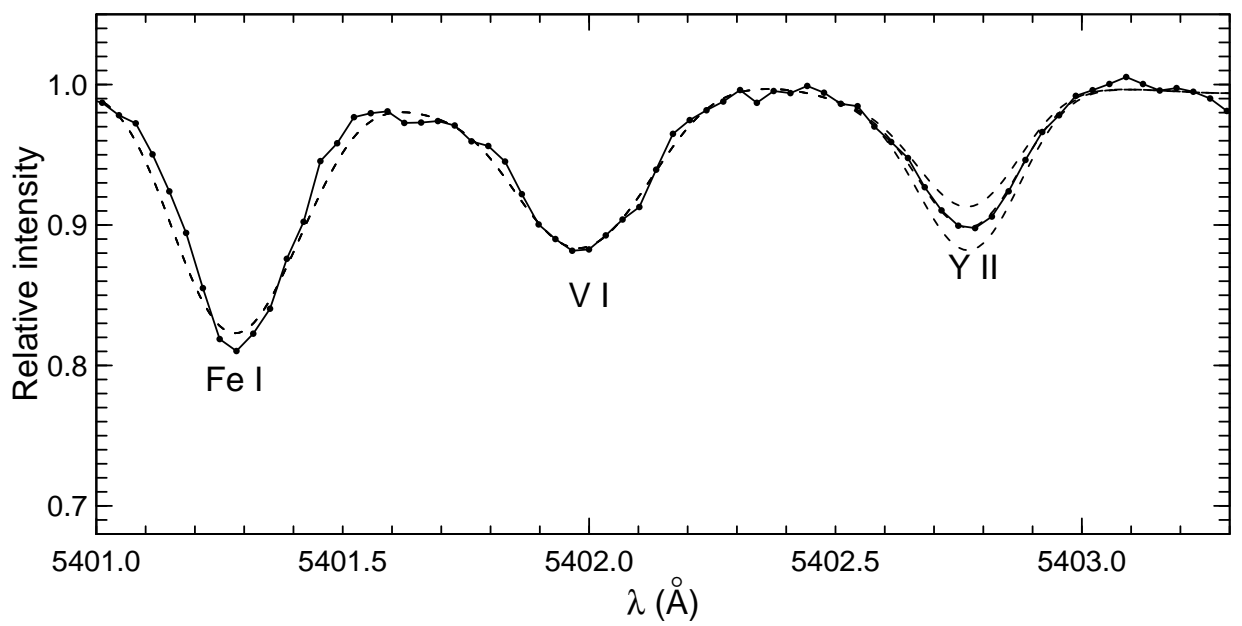

Fig. 5. Synthetic spectrum fit to the Y II line at $5402.78 \AA$ for $\lambda$ And. The observed spectrum is shown as the solid line joining the dots. The dashed lines denote the synthetic spectra with $[\mathrm{Y} / \mathrm{Fe}]=0.05,0.15$ and 0.25 dex downward.

\subsection{Estimation of uncertainties}

Sources of errors in our research can be divided into two distinct categories. The first one includes the errors that affect only single lines (e.g., the errors in equivalent width measurements or line parameters). Other sources of observational errors, such as the continuum placement or background subtraction problems, also are partly included in the equivalent width errors. The scatter of the deduced line abundances, $\sigma$, presented in Table 3 , gives an estimation of the uncertainty due to random errors in the line parameters (the mean value of $\sigma$ is $0.05 \mathrm{dex}$ ). Thus 


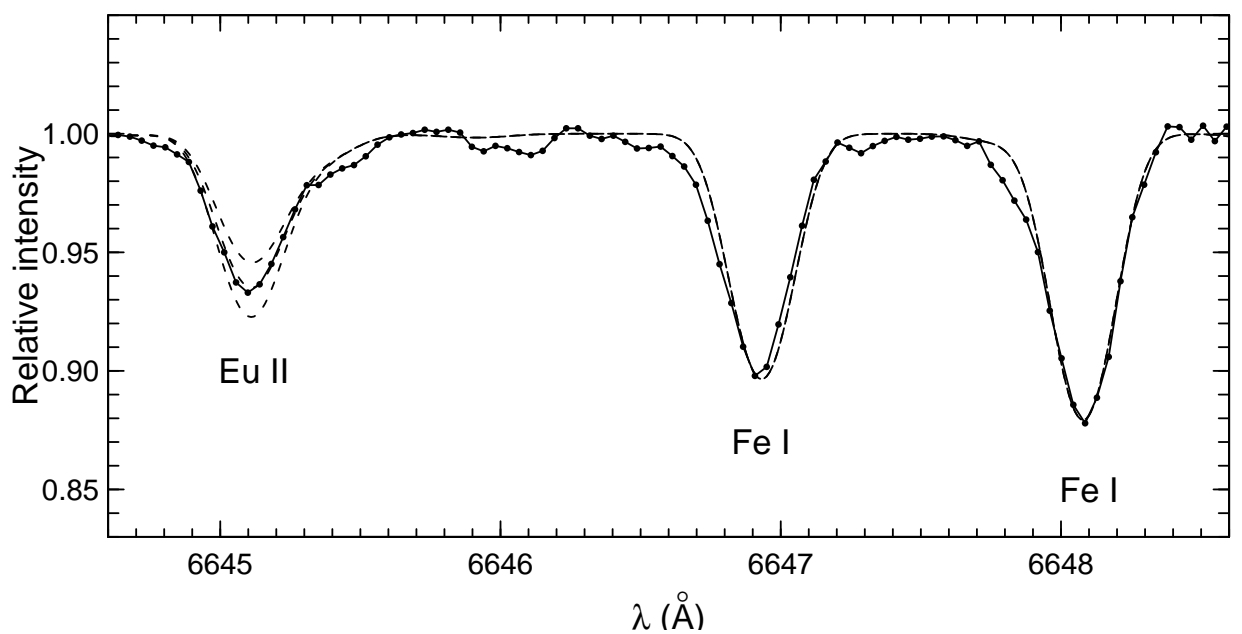

Fig. 6. Synthetic spectrum fit to the Eu II line at $6645.10 \AA$ for $\lambda$ And. The observed spectrum is shown as the solid line joining the dots. The dashed lines denote the synthetic spectra with $[\mathrm{Eu} / \mathrm{Fe}]=0.28,0.38$ and 0.48 dex downward.

the uncertainties in the derived abundances that are the result of random errors are close to this value.

The second category includes the errors which affect all the lines together. These are mainly the model errors, such as errors in the effective temperature, surface gravity, microturbulent velocity, etc.). The sensitivity of the abundance estimates to changes in the atmospheric parameters by the assumed errors is illustrated in Table 2.

Since the abundances of $\mathrm{C}, \mathrm{N}$ and $\mathrm{O}$ are bound together by the molecular equilibrium in the stellar atmosphere, we have also investigated how the error in one of them typically affects the abundance determination of another. $\Delta[\mathrm{O} / \mathrm{H}]=$ 0.10 causes $\Delta[\mathrm{C} / \mathrm{H}]=0.04$ and $\Delta[\mathrm{N} / \mathrm{H}]=0.03 ; \Delta[\mathrm{C} / \mathrm{H}]=0.10$ causes $\Delta[\mathrm{N} / \mathrm{H}]=$ -0.12 and $\Delta[\mathrm{O} / \mathrm{H}]=0.03 . \Delta[\mathrm{N} / \mathrm{H}]=0.10$ has no effect on either the carbon or the oxygen abundances.

\section{RESULTS AND DISCUSSION}

\subsection{Atmospheric parameters and the iron abundance}

As a result, for $\lambda$ And we have determined the following atmospheric parameters: $T_{\text {eff }}=4830 \mathrm{~K}, \log g=2.8, v_{\mathrm{t}}=1.6 \mathrm{~km} \mathrm{~s}^{-1},[\mathrm{Fe} / \mathrm{H}]=-0.53,[\mathrm{C} / \mathrm{Fe}]=0.09$, $[\mathrm{N} / \mathrm{Fe}]=0.35,[\mathrm{O} / \mathrm{Fe}]=0.45, \mathrm{C} / \mathrm{N}=2.21,{ }^{12} \mathrm{C} /{ }^{13} \mathrm{C}=14$. The element abundances $[\mathrm{A} / \mathrm{H}]$ and $\sigma$ (the line-to-line scatter) are listed in Table 3 and compared with results of other investigations in Figure 7.

$\lambda$ And has a long history of high resolution spectral investigations. The first were Helfer \& Wallerstein (1968) who studied the star by the differential curveof-growth method. The chemical composition of $\lambda$ And by modern methods using stellar atmosphere models was investigated by McWilliam (1990), Tautvaišienė et al. (1992), Savanov \& Berdyugina (1994), Donati et al. (1995). The lithium abundance in $\lambda$ And was investigated by Randich et al. (1994) and Mallik (1998) 
Table 2. The sensitivity of abundances to the changes in the atmospheric parameters. The table entries show the effects on the logarithmic abundance relative to hydrogen, $\Delta[\mathrm{A} / \mathrm{H}]$.

\begin{tabular}{|c|c|c|c|c|c|c|c|}
\hline Element & $\begin{aligned} & \Delta T_{\text {eff }} \\
+ & 100 \mathrm{~K}\end{aligned}$ & $\begin{array}{c}\Delta \log g \\
+0.3\end{array}$ & $\begin{array}{c}\Delta v_{\mathrm{t}} \\
+0.3 \mathrm{~km} / \mathrm{s}\end{array}$ & Element & $\begin{aligned} & \Delta T_{\text {eff }} \\
+ & 100 \mathrm{~K}\end{aligned}$ & $\begin{array}{c}\Delta \log g \\
+0.3\end{array}$ & $\begin{array}{c}\Delta v_{\mathrm{t}} \\
+0.3 \mathrm{~km} / \mathrm{s}\end{array}$ \\
\hline $\mathrm{C}\left(\mathrm{C}_{2}\right)$ & -0.01 & 0.01 & 0.10 & Fe II & -0.07 & 0.15 & -0.03 \\
\hline $\mathrm{N}(\mathrm{CN})$ & 0.07 & 0.00 & 0.09 & Co I & 0.08 & 0.05 & -0.03 \\
\hline $\mathrm{O}([\mathrm{O} \mathrm{I}])$ & 0.01 & 0.01 & 0.14 & $\mathrm{Ni}$ I & 0.04 & 0.05 & -0.07 \\
\hline $\mathrm{Na} \mathrm{I}$ & 0.15 & -0.01 & -0.01 & Y II & 0.00 & 0.12 & -0.01 \\
\hline Si I & -0.02 & -0.06 & -0.02 & $\mathrm{ZrI}$ & 0.17 & 0.00 & -0.02 \\
\hline Ca I & 0.09 & 0.00 & -0.05 & La II & 0.01 & 0.12 & -0.01 \\
\hline Sc II & -0.01 & 0.12 & -0.09 & Ce II & 0.01 & 0.12 & -0.01 \\
\hline Ti I & 0.12 & 0.00 & -0.07 & Pr II & 0.02 & 0.13 & -0.01 \\
\hline VI & 0.15 & 0.01 & -0.07 & Nd II & 0.01 & 0.12 & -0.01 \\
\hline Cr I & 0.09 & 0.00 & -0.08 & Eu II & -0.01 & 0.13 & 0.00 \\
\hline $\mathrm{Fe} I$ & 0.06 & 0.03 & -0.04 & & & & \\
\hline $\mathrm{C} / \mathrm{N}$ & -0.28 & 0.06 & 0.06 & ${ }^{12} \mathrm{C} /{ }^{13} \mathrm{C}$ & 0.00 & 0.00 & 1.00 \\
\hline
\end{tabular}

Table 3. Element abundances relative to hydrogen $[\mathrm{A} / \mathrm{H}] . \sigma$ is a standard deviation in the mean value determined from the line-to-line scatter within the species. $N$ is the number of lines used for the abundance determination.

\begin{tabular}{|c|c|c|c|c|c|c|c|}
\hline Element & $N$ & {$[\mathrm{El} / \mathrm{H}]$} & $\sigma$ & Element & $N$ & {$[\mathrm{El} / \mathrm{H}]$} & $\sigma$ \\
\hline $\mathrm{C}\left(\mathrm{C}_{2}\right)$ & 1 & -0.44 & - & Fe II & 8 & -0.54 & 0.07 \\
\hline $\mathrm{N}(\mathrm{CN})$ & 5 & -0.18 & 0.04 & Co I & 4 & -0.43 & 0.07 \\
\hline $\mathrm{O}([\mathrm{O} \mathrm{I}])$ & 1 & -0.08 & - & $\mathrm{Ni}$ I & 19 & -0.54 & 0.04 \\
\hline $\mathrm{NaI}$ & 1 & -0.22 & - & Y II & 1 & -0.38 & - \\
\hline Si I & 2 & -0.31 & 0.03 & $\mathrm{Zr} \mathrm{I}$ & 3 & -0.17 & 0.11 \\
\hline $\mathrm{Ca} \mathrm{I}$ & 3 & -0.10 & 0.05 & La II & 1 & -0.37 & - \\
\hline Sc II & 2 & -0.26 & 0.05 & Ce II & 2 & -0.51 & 0.03 \\
\hline Ti I & 16 & -0.01 & 0.04 & Pr II & 1 & -0.15 & - \\
\hline V I & 15 & -0.04 & 0.05 & $\mathrm{Nd}$ II & 1 & -0.15 & - \\
\hline Cr I & 5 & -0.37 & 0.07 & Eu II & 1 & -0.15 & - \\
\hline $\mathrm{Fe} I$ & 44 & -0.53 & 0.03 & & & & \\
\hline
\end{tabular}

and just the main atmospheric parameters recently were determined by Soubiran et al. (2008).

The available values of the effective temperature for $\lambda$ And cover a wide interval, from $4032 \mathrm{~K}$ (Helfer \& Wallerstein 1968) to $4850 \mathrm{~K}$ (Randich et al. 1994). Our result $(4830 \mathrm{~K})$ is in good agreement with the Randich et al. determination. The temperature determined by Donati et al. (1995) is by $80 \mathrm{~K}$ lower, but this does not exceed the temperature determination errors.

The values of surface gravities $(\log g)$ for $\lambda$ And are in the interval from 2.0 (Tautvaišienè et al. 1992) to 3.1 (McWilliam 1990; Mallik 1998; Soubiran et al. 2008). Our present value $(\log g=2.8)$ is in the best agreement with that of Randich et al. (1994); the differences with the results of other studies are also within the error box.

The $[\mathrm{Fe} / \mathrm{H}]$ values, received for $\lambda$ And, extend from -0.43 (Mallik 1998) to -0.80 (Tautvaišienè et al. 1992). The average value of $[\mathrm{Fe} / \mathrm{H}]$ of the previous studies is -0.58 . Our value, $[\mathrm{Fe} / \mathrm{H}]=-0.53$, is close to this average. 


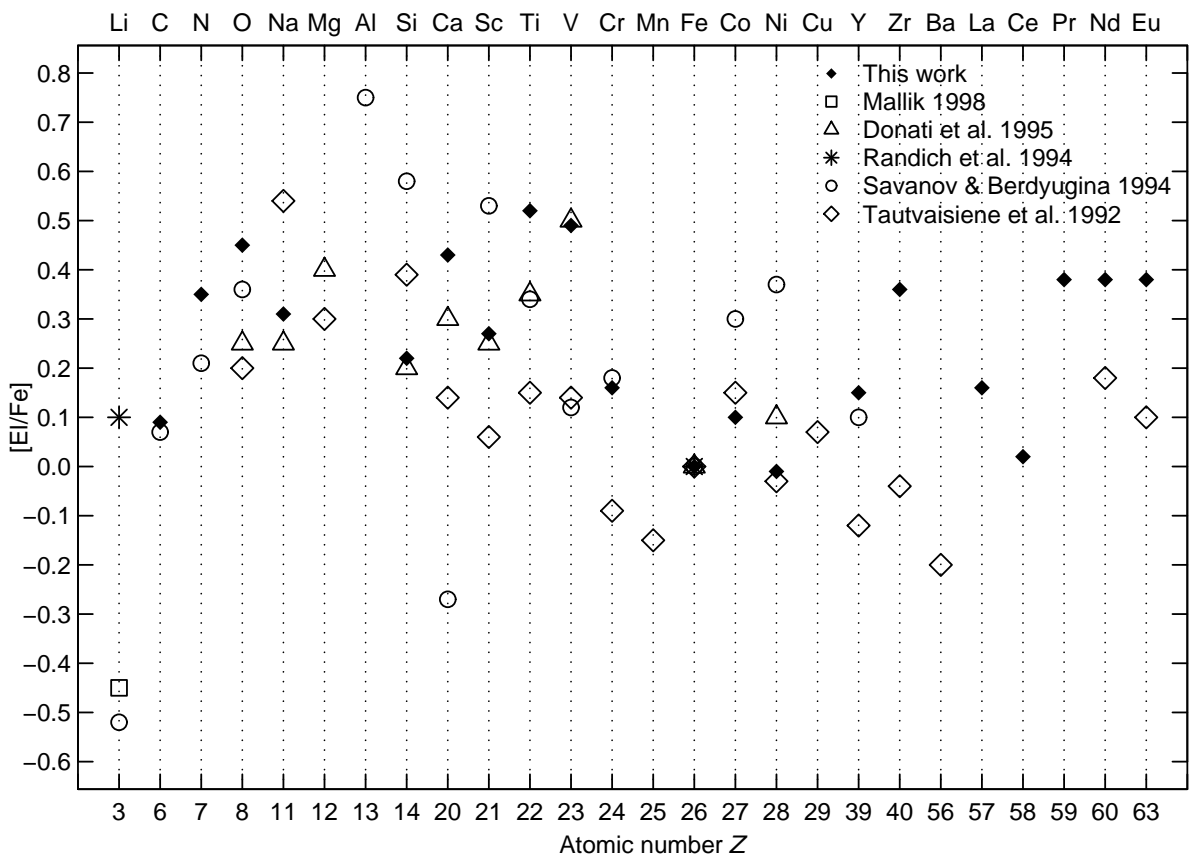

Fig. 7. Abundances of elements for $\lambda$ And, as determined in this work (filled diamonds), Donati et al. (1995, triangles), Savanov \& Berdyugina (1994, circles), Tautvaišienè et al. (1992, empty diamonds). The [Li/Fe] value from Randich et al. (1994) is shown as an asterisk and from Mallik (1998) as a square.

\subsection{Carbon and nitrogen}

For the interpretation of carbon and nitrogen abundances in $\lambda$ And, we have to remind several evolutionary episodes of low-mass red giants.

The first opportunity for low-mass stars to modify their surface carbon and nitrogen abundances happens on their way to the red giant branch when they undergo the so-called first dredge-up (Iben 1965). During this evolutionary stage, the deepening convective envelope mixes the outer layers of the red giant with the internal matter which has been $\mathrm{CN}$-processed while the star resided on the main sequence. Convective mixing induces a change of the carbon and nitrogen surface abundances. The atmospheric abundance of ${ }^{12} \mathrm{C}$ decreases, while the ${ }^{13} \mathrm{C}$ and ${ }^{14} \mathrm{~N}$ abundances increase.

However, the classical stellar evolution theory considers stars as non-rotating and non-magnetic bodies, and the convection is accepted as the only mixing process. Observations of $\mathrm{CNO}$ elements in a large number of evolved low-mass giants show much lower $\mathrm{C} / \mathrm{N}$ and ${ }^{12} \mathrm{C} /{ }^{13} \mathrm{C}$ ratios than the post-dredge-up ratios predicted in the framework of standard stellar theory (see reviews by Chanamé et al. 2005 and Charbonnel 2006). Therefore, it was concluded that the next distinct mixing episode in low-mass stars occurs at the so-called red giant branch 'bump'. It was surmised that at this evolutionary step the mean molecular weight gradient, produced by the first dredge-up and inhibiting mixing processes, is erased by the outwardly-burning hydrogen shell and results in extra mixing of the convective zone material with regions hot enough to convert ${ }^{12} \mathrm{C}$ to ${ }^{13} \mathrm{C}$. This evolutionary 
phase is referred to as the 'bump' of the luminosity function on the HR diagram and it corresponds to a temporary decrease in the luminosity and a small increase in the effective temperature of the star when the chemical discontinuity is removed.

In Figure 8 we compare $\mathrm{C} / \mathrm{N}$ and ${ }^{12} \mathrm{C} /{ }^{13} \mathrm{C}$ ratios of $\lambda$ And with two theoretical models of extra-mixing. The first model, called as 'cool bottom processing' (CBP), was calculated by Boothroyd \& Sackmann (1999). It includes the deep circulation mixing below the base of the standard convective envelope and the consequent processing of $\mathrm{CNO}$ isotopes.
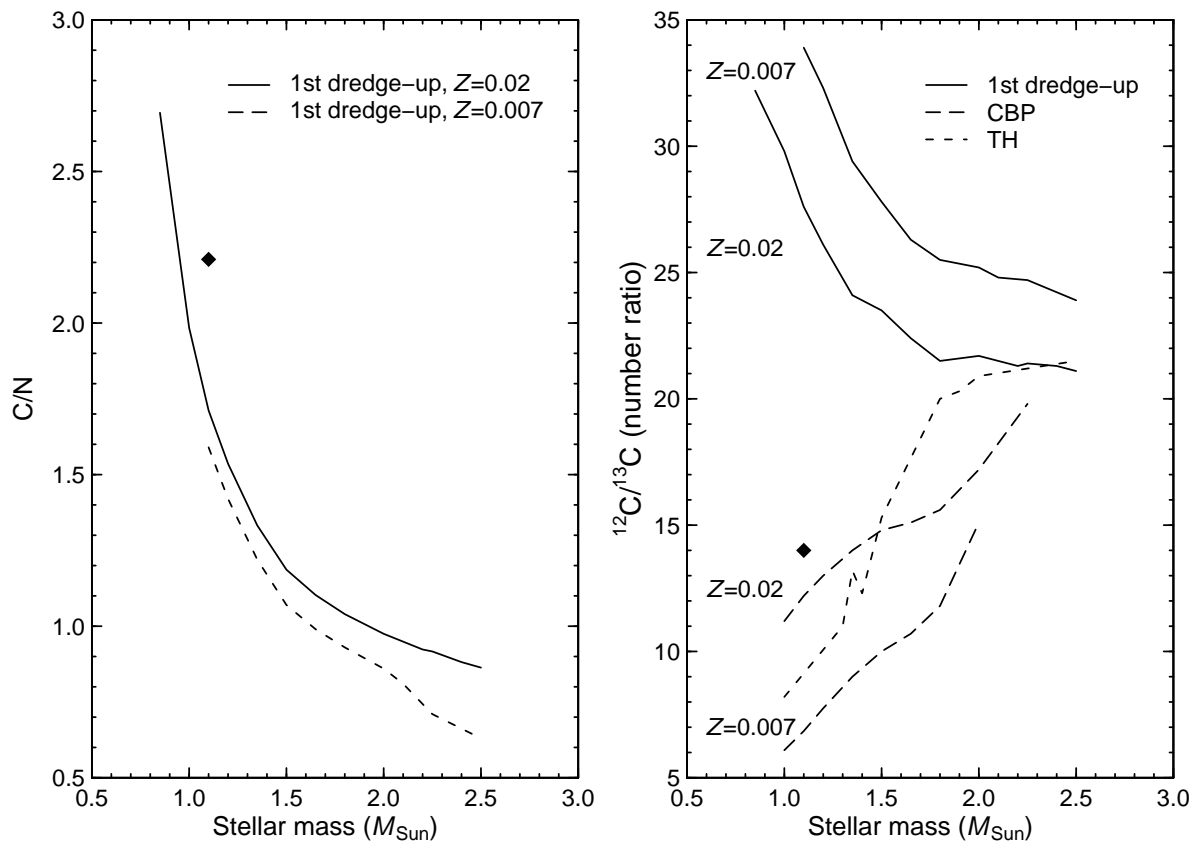

Fig. 8. Comparisons of $\mathrm{C} / \mathrm{N}$ and ${ }^{12} \mathrm{C} /{ }^{13} \mathrm{C}$ ratios of $\lambda$ And (diamonds) with the theoretical predictions for two metallicities. Left panel: the $\mathrm{C} / \mathrm{N}$ values in the first dredge-up (Boothroyd \& Sackman 1999). Right panel: the ${ }^{12} \mathrm{C} /{ }^{13} \mathrm{C}$ values for the first dredge-up and the cool bottom processing (CBP) model by Boothroyd \& Sackman (1999) and the model of thermohaline mixing (TH) by Charbonnel \& Lagarde (2010).

Another model, called as the 'thermohaline mixing' (TH), was recently presented by Charbonnel \& Lagarde (2010). Following Eggleton et al. (2006) and Charbonnel \& Zahn (2007), this model considers a double diffusive instability referred to as thermohaline convection, which had been discussed long ago in the literature (Stern 1960), as an important process in evolution of red giants. This mixing connects the convective envelope with the external wing of hydrogen burning shell and induces surface abundance modifications in red giant stars. The mean molecular weight inversion at the red giant bump is produced by the reaction ${ }^{3} \mathrm{He}\left({ }^{3} \mathrm{He}, 2 \mathrm{p}\right){ }^{4} \mathrm{He}$, as predicted by Ulrich (1972). According to the authors, the thermohaline mixing does not occur earlier than the bump, since the magnitude of the mean molecular wight inversion is small compared to a stabilizing mean molecular weight stratification.

According to the comparison with stellar evolutionary sequences in the lumi- 
nosity versus effective temperature diagram by Girardi et al. (2000), $\lambda$ And with its luminosity $\log \left(L / L_{\odot}\right)=1.37$ is a first ascent giant lying slightly below the red giant sequence bump indicated at $\log \left(L / L_{\odot}\right)=1.6$ (Charbonnel \& Lagarde 2010). According to the mentioned models of mixing, carbon and nitrogen abundances should be altered only by the first dredge-up.

However, the position of $\lambda$ And in the ${ }^{12} \mathrm{C} /{ }^{13} \mathrm{C}$ versus stellar mass diagram (Figure 8 ) indicates that its carbon isotope ratio is altered by extra mixing. The low value of the ${ }^{12} \mathrm{C} /{ }^{13} \mathrm{C}$ ratio in $\lambda$ And gives a hint that extra-mixing processes may start acting in low-mass chromospherically active stars below the currently predicted place of the bump of the luminosity function of red giants. It is interesting that the $\mathrm{C} / \mathrm{N}$ ratio of $\lambda$ And is similar to that of II Peg, another very active RS CVn-type star with low metallicity $([\mathrm{Fe} / \mathrm{H}]=-0.4, \mathrm{C} / \mathrm{N} \sim 2$, Berdyugina et al. 1998a). However, the ${ }^{12} \mathrm{C} /{ }^{13} \mathrm{C}$ ratio of II Peg is not known, and this prevents evaluation whether this star has undergone additional mixing. This is an important task for future.

\subsection{Other heavy elements}

Abundance ratios of iron peak elements investigated in this work $(\mathrm{Cr}, \mathrm{Co}, \mathrm{Ni}$ and $\mathrm{Sc}$ ) to the abundance of $\mathrm{Fe}$ are close to the solar ratio. Only vanadium is overabundant by $[\mathrm{V} / \mathrm{Fe}]=0.5$. The same value was obtained by Donati et al. (1995); most probably the overabundance is caused by the hyperfine splitting effects. However, Savanov \& Berdyugina (1994) and Tautvaišien e et al. (1992) have received almost solar ratios. Donati et al. (1995), applying 11 spectrograms of $\lambda$ And taken in 1991 and 1994, investigated the dependence of equivalent widths of lines on the rotational phase and the surface spot coverage. They have noticed that for some lines, e.g., Fe I, the equivalent widths remain almost unchanged in the presence of cool spots. However, the lines of V I and Ti I increase noticeably. Our results show that $[\mathrm{El} / \mathrm{Fe}]$ ratios are the largest for these particular elements.

We obtained the following average values of abundances for $\alpha^{-}$, s- and relements: $[\alpha / \mathrm{Fe}]=0.4,[\mathrm{~s} / \mathrm{Fe}]=0.2$ and $[\mathrm{r} / \mathrm{Fe}]=0.4$. These ratios are by $0.2-0.25$ dex lower than the those of the theoretical models calculated for the thin disk of the Milky Way by Pagel \& Tautvaišienè $(1995,1997)$ at the metallicity -0.5 dex. Similar overabundances of $\alpha$-elements were obtained in a sample of other RS CVn stars investigated by Morel et al. (2004). From the s- and r-process elements, only barium has been investigated by Morel et al. and found to be also overabundant.

Abundances of $\mathrm{Mg}$ and $\mathrm{Al}$ were not investigated in our work. As it is seen from Figure 7, the available $[\mathrm{Mg} / \mathrm{Fe}]$ values (Donati et al. 1995; Tautvaišienè et al. $1992)$ are in agreement with other $\alpha$-elements. Aluminum was investigated only by Savanov \& Berdyugina (1994) and found to be overabundant even by 0.7 dex. We also did not investigate the abundance of lithium. The available [Li/Fe] values for $\lambda$ And are quite different. Savanov \& Berdyugina (1994) and Mallik (1998) find about -0.5 dex, while the result of Randich et al. (1994) is +0.1 dex.

More observational and theoretical studies of RS CVn stars are essential in trying to answer the questions of the role of magnetic fields in stellar plasma dynamics, the relation between the stellar structure and energy balance, the interaction between stellar rotation and orbital motion.

ACKNOWLEDGMENTS. This project was supported by the European Commission through the Baltic Grid project. 


\section{REFERENCES}

Alekseev I. Yu., Kozhevnikova A. V. 2005, Astrophysics, 48, 450

Alonso A., Arribas S., Martínez-Roger C. 1999, A\&AS, 140, 261

Anstee S. D., O’Mara B.J. 1995, MNRAS, 276, 859

Audard M., Güdel M., Sres A., Mewe R., Raassen A. A. J., Behar E., Foley C. R., van der Meer R. L. J. 2001, in Stellar Coronae in the Chandra and XMMNewton Era, eds. F. Favata \& J. J. Drake, ASP Conf. Ser., 277, 65

Audard M., Güdel M., Sres A., Mewe R., Raassen A. A. J., Behar E., Mewe R. 2003, A\&A, 398, 1137

Barklem P. S., O’Mara B.J. 1997, MNRAS, 290, 102

Barklem P. S., O’Mara B.J., Ross J.E. 1998, MNRAS, 296, 1057

Berdyugina S. V. 2005, Living Rev. Solar Phys., 2, 8

Berdyugina S. V., Jankov S., Ilyin I., Tuominen I., Fekel F. C. 1998a, A\&A, 334, 863

Berdyugina S. V., Berdyugin A. V., Tuominen I., Fekel F. C. 1998b, A\&A, 340, 437

Berdyugina S. V., Ilyin I., Tuominen I. 1999, A\&A, 347, 932

Berdyugina S. V., Berdyugin A. V., Ilyin I., Tuominen I. 2000, A\&A, 360, 272

Biehl D. 1976, Diplomarbeit, Christian-Albrechts-Universität Kiel, Institut für Theoretishe Physik und Sternwarte

Boothroyd A. I., Sackman I. J. 1999, ApJ, 510, 232

Bopp B. W., Noah P. V. 1980, PASP, 92, 717

Bowyer S., Drake J. J., Vennes S. 2000, ARA\&A, 38, 231

Calder W. A. 1938, Bull. Harvard Obs., 907, 20

Chanamé J., Pinsonneault M., Terndrup D. 2005, ApJ, 631, 540

Charbonnel C. 2006, in Stars and Nuclei: Tribute to Manuel Forestini, eds. T. Montmerle \& C. Kahane, EAS Publ. Ser., 19, 125

Charbonnel C., Lagarde N. 2010, A\&A, accepted for publication (arXiv1006.5359)

Charbonnel C., Zahn J.-P. 2007, A\&A, 467, L15

Chisari D., Lacona G. 1965, Mem. Soc. Astron. Ital., 36, 463

De Medeiros J. R., Da Silva J. R. P., Maia M. R. G. 2002, ApJ, 578, 943

Donati J.-F., Henry G. W., Hall D. S. 1995, A\&A, 293, 107

Drake J. J. 1996, in Cool Stars, Stellar Systems and the Sun, 9th Cambridge Workshop, eds. R. Pallavicini \& A. K. Dupree, ASP Conf. Ser., p. 203

Drake J. J. 2002, in Stellar Coronae in the Chandra and XMM-Newton Era, eds. F. Favata \& J. J. Drake, ASP Conf. Ser., 277, 75

Eggleton P. P., Dearborn D. S. P., Lattanzio J. C. 2006, Science, 314, 1580

Frasca A., Biazzo K., Tas G., Evren S., Lanzafame A. C. 2008, A\&A, 479, 557

Girardi L., Bressan A., Bertelli G., Chiosi C. 2000, A\&AS, 141, 371

Gondoin P. 2007, A\&A, 464, 1101

Gonzalez G., Lambert D. L., Wallerstein G. et al. 1998, ApJS, 114, 133

Grevesse N., Sauval A. J. 2000, in Origin of Elements in the Solar System, Implications of Post-1957 Observations, ed. O. Manuel, Kluwer, p. 261

Gurtovenko E. A., Kostik R. I. 1989, in Fraunhofers spectrum and a System of Solar Oscillator Strengths, Naukova Dumka, Kyiv, Ukraine

Gustafsson B., Edvardsson B., Eriksson K., Jørgensen U. G., Nordlund Å., Plez B. 2008, A\&A, 486, 951

Hakkila J., Myers J. M., Stidham B. J., Hartmann D. H. 1997, AJ, 114, 2043

Hall D. S. 1976, in Structure and Evolution of Close Binary Systems, eds. P. Eggleton, S. Mitton \& J. Whelan, IAU Symp. 73, p. 381

Hauck B., Mermilliod M. 1998, A\&AS, 129, 431 
Helfer H. L., Wallerstein G. 1968, ApJS, 16, 1

Iben I. 1965, ApJ, 142, 1447

Ilyin I. V. 2000, in High Resolution SOFIN CCD Echelle Spectroscopy, PhD thesis, University of Oulu, Finland, 266 p.

Jeffers S. V. 2005, MNRAS, 359, 729

Johansson S., Litzen U., Lundberg H., Zhang Z. 2003, ApJ, 584, 107

Jordan C., Doschek G. A., Drake J. J., Galvin A. B., Raymond J. C. 1998, in The Tenth Cambridge Workshop on Cool Stars, Stellar Systems and the Sun, ASP Conf. Ser. 154, 91

Katz D., Favata F., Aigrain S., Micela G. 2003, A\&A, 397, 747

Kurucz R.L. 2005, New Atlases for Solar Flux, Irradiance, Central Intensity and Limb Intensity, Mem. Soc. Astron. Ital. Suppl., 8, 189

Lawler J. E., Wickliffe M. E., Den Hartog E. A. 2001, ApJ, 563, 1075

Mallik S. V. 1998, A\&A, 338, 623

Mäckle R., Holweger H., Griffin R., Griffin R. 1975, A\&A, 38, 239

McWilliam A. 1990, ApJS, 74, 1075

Mirtorabi M. T., Wasatonic R., Guinan E. F. 2003, AJ, 125, 3265

Montesinos B., Giménez A., Fernández-Figueroa M. J. 1988, MNRAS, 232, 361

Morel T., Micela G., Favata F., Katz D., Pillitteri I. 2003, A\&A, 412, 495

Morel T., Micela G., Favata F., Katz D., Pillitteri I. 2004, A\&A, 426, 1007

Olivier J. P. 1974, PASP, 87, 695

O’Neal D., Neff J. E., Saar S. H. 1998, ApJ, 507, 919

Padmakar, Pandey S. K. 1999, A\&AS, 138, 203

Pagel B. E. J., Tautvaišienè G. 1995, MNRAS, 276, 505

Pagel B. E. J., Tautvaišienè G. 1997, MNRAS, 288, 108

Pallavicini R., Randich S., Giampapa M.S. 1992, A\&A, 253, 185

Piskunov N. E., Kupka F., Ryabchikova T. A., Weiss W. W., Jeffery C. S. 1995, A\&A, 112,525

Poe C. H., Eaton J. A. 1985, ApJ, 289, 644

Randich S., Gratton R., Pallavichini R. 1993, A\&A, 273, 194

Randich S., Giampapa M. S., Pallavichini R. 1994, A\&A, 283, 893

Rodonó M. 1965, PhD Thesis, University of Catania, Italy

Rodonó M., Lanza A. F., Catalano S. 1995, A\&A, 301, 75

Sanz-Forcada J., Favata F., Micela G. 2004, A\&A, 416, 281

Savanov I. S., Berdyugina S. V. 1994, Astronomy Letters, 20, 227

Simmons G. J., Blackwell D. E. 1982, A\&A, 112, 209

Soubiran C., Bienaymé O., Mishenina T., Kovtyukh V. V. 2008, A\&A, 480, 91

Stern M. E. 1960, Tellus, 12, 172

Strassmeier K. G., Briguglio R., Granzer T., Tosti G. et al. 2008, A\&A, 490, 287

Tautvaišienè G., Kriukelis S., Bikmaev I. 1992, Baltic Astronomy, 1, 450

Uesugi A., Fukuda I. 1982, Catalogue of Stellar Rotational Velocities, Kyoto University

Ulrich R. K. 1972, ApJ, 172, 165

Unsöld A. 1955, Physik der Stern Atmosphären, Zweite Auflage, Springer-Verlag, Berlin

van Leeuwen F. 2007, Hipparcos, the New Reduction of the Raw Data, Astrophysics and Space Science Library, Vol. 350

Walker E. C. 1944, JRASC, 38, 249

Zahn J.-P. 1977, A\&A, 57, 383 